\title{
Mitochondrial DNA Sequence and Gene Organization in the Australian Blacklip Abalone Haliotis rubra (Leach)
}

Ben T. Maynard, Lyndal J. Kerr, Joanne M. McKiernan, Eliza S. Jansen, Peter J. Hanna

The above article (DOI: 10.1007/s10126-005-0013-z) published online with typographical errors in the title. It appears correctly above and in the print issue (Vol. 7, No. 6). The publisher apologizes for the error. 